\title{
A meshless method solution approach for the transient poro-elastic levee problem
}

\author{
E. Divo ${ }^{1} \&$ A. Kassab ${ }^{2}$ \\ ${ }^{1}$ Engineering Technology, Daytona State College, USA \\ ${ }^{2}$ Mechanical, Materials and Aerospace Engineering Department, \\ University of Central Florida, USA
}

\begin{abstract}
We apply the localized collocation Meshless method (LCCM) to the solution of the problem of poro-elastic flow in a levee. In this approach the interaction between the water flow and soil is resolved in a time-accurate manner. Keywords: meshless methods, poro-elasticity.
\end{abstract}

\section{Introduction}

Water retaining structures, such as dams, canals, and levees, are essential for managing water resources. Building them with locally available soils is often the most economical, environmentally friendly, and sustainable method. It is possible sometimes to economically construct these structures with masonry, concrete, or even alternative materials. However, they must be placed on highquality foundation soils for satisfactory performance wherever a solid rock stratum is not available at a reasonable depth. Therefore, the safety of most of these hydraulic structures depends on the capacity of the soils in the body and/or the foundation to resist the seepage forces created by the retained water. In other words, the soils should be able to safely seepage without damage or erosion of soils.

Often times, the seepage on the downstream sides of hydraulic structures is found to be laden with soil particles, a phenomenon commonly referred to as "sand boils". Excessive seepage at specific points on the downstream side is indicative of preferential flow that may be due to anomalies in construction, foundation strata, or formation of internal voids due to movement of soils under excessive seepage forces. Formation and gradual expansion of internal voids is 
frequently referred to as "progressive piping". This phenomenon may proceed without getting noticed as it occurs internally deep within the structure. The internal voids may collapse and form surface sinkholes, and in some cases may rapidly cause breaching failure of the structure. ICOLD [11] and Foster et al. [6] conducted statistical analyses of dam failures and found that internal soil erosion is a predominant cause of failure.

External forces caused by differential settlements, reservoir level fluctuations, and geological disturbances result in the formation of tiny internal cracks whose orientation and dimensions may vary widely owing to the differences in the magnitudes and directions of those forces. These tiny cracks may enlarge into large pipes depending on the severity of hydraulic and mechanical conditions in their vicinity. A fundamental consideration of these conditions is essential for a better understanding of the mechanism of progressive piping. Zaslavsky and Kassiff [26], Vallejo [23], and Kakuturu and Reddi [15] have described these conditions and outlined some basic methods for their assessment. This paper presents an advanced numerical approach and demonstrates its applicability for analyzing the pore water pressures, hydraulic gradients and principal stresses around such cracks in earth dams.

\section{Background}

The first reservoir filling is considered as very crucial because soils might shrink and develop desiccation cracks during the period between construction and reservoir filling, and the stresses produced might enlarge the cracks. Zhang and Chen [27] reported their analysis of the seepage failure mechanism of a 71-m high Chinese rockfill dam that failed catastrophically during its first reservoir filling in 1993. During service life, cracks may develop, particularly during intermittent dry periods that also witness differential settlements of structural components caused by the compression of the foundation, or even by minor unnoticed earthquakes. Lakshmikantha et al. [16] conducted laboratory experiments on clayey soils and employed image analysis techniques for understanding the pattern of cracks and the internal and boundary conditions that influence them.

A few examples of piping-induced major failures are that of Teton Dam described by Watts et al. [24], Chagrin River Dam in Ohio analyzed by Evans et al. [5], in addition to several cases described in ICOLD [11]. The Katrina disaster in Louisiana and frequent problems faced by levees along the Mississippi, Sacramento, and Red rivers have heightened the public concerns. Mansur et al. [17] reported the sand boils caused by piping along the Mississippi river. Sills et al. [22] inferred that piping was the probable reason for failure of some levees in New Orleans that were not overtopped during the Katrina hurricane. Hagerty [8, 9] provide a comprehensive and visual description of conditions that result in piping and methods for post-failure identification. Gattinoni and Francani [7] developed a model for studying the instability triggered by piping and applied it to the Stava Valley disaster that took place in Italy, 1985. 
Johansen and Eikevik [13] described the internal erosion suffered by the Jukla Dams in Norway, exhuming operations that confirmed piping, and the rehabilitation measures. The internal erosion and progressive piping was detected in several experimental investigations of problem-riddled levees and dams. Studies by Chen et al. [3], Sjödahl [21], Inazaki [12], and Hung et al. [10] relied upon non-invasive geophysical methods that provided qualitative evidence of progressive piping.

Though thousands of tiny cracks may develop in a hydraulic structure during a year, most of them may self-heal with only a very few having a likelihood of growing larger and result in progressive piping. Developing a mechanistic understanding of progressive piping has been undertaken by Sellmeijer and Koenders [20], Ojha et al. [18], Kakuturu and Reddi [15], Bonelli et al. [1], El Shamy and Aydin [4], and Bonelli and Brivois [2] while recent research by Ojka et al. [18], and Yi et al. [25] has focused on the transient nature of piping. Kakuturu and Reddi [14] have described the experimental methods for evaluation of some soil properties that influence progressive piping.

It can be summarized that the pore water pressures, hydraulic gradients, and principal stresses in the region surrounding the tiny cracks are the prime external drivers of progressive piping. These factors in conjunction with soil properties, viz., shear strength, gradation, interlocking, cohesion, particle transportability, pore water chemistry, govern the fate of tiny cracks. In this paper, we adopt a numerical approach based on a novel Localized Collocation Meshless Method (LCMM) [28-32] for studying the fate of cracks under transient loading of a levee due to variable winds, storm surges, and cyclic variations of reservoir levels.

\section{The governing equations of poro-elasticity}

The equations that govern the flow of a fluid through a poro-elastic medium are the Navier equation coupled with the Richards equation [1] as:

$$
\begin{aligned}
& G \nabla^{2} \vec{u}+\frac{G}{(1-2 \nu)} \nabla(\nabla \cdot \vec{u})=\nabla p \\
& \phi \beta_{f} \frac{\partial p}{\partial t}=\nabla \cdot\left[\frac{\kappa}{\mu_{f}}\left(\nabla p+\rho_{f} \vec{g}\right)\right]-\frac{\partial}{\partial t}(\nabla \cdot \vec{u})
\end{aligned}
$$

Here, $p$ is the pore pressure, $\phi$ is the porosity of the medium, $\kappa$ is the permeability of the medium, and $\beta_{f}, \rho_{f}, \mu_{f}$ are respectively the compressibility, density, and viscosity of the fluid flowing through the porous medium. Notice that the two governing equations in (1) are strongly coupled (two-way) as the pore pressure appears on the right-hand side of the Navier equation while the deformation field dilatation, $(\nabla \cdot \vec{u})$, appears on the right-hand side of the Richards equation. An important parameter in flow through porous media is the hydraulic conductivity which is defined as:

$$
K_{h}=\frac{\kappa \rho_{f}|\vec{g}|}{\mu_{f}}
$$


The Darcy velocity of the flow through the porous medium is defined as:

$$
\vec{V}=-\frac{\kappa}{\mu_{f}}\left(\nabla p+\rho_{f} \vec{g}\right)
$$

And, therefore, the actual front velocity of the flow is given by:

$$
\vec{V}_{f}=\frac{\partial \vec{u}}{\partial t}-\frac{\kappa}{\phi \mu_{f}}\left(\nabla p+\rho_{f} \vec{g}\right)
$$

The front velocity of the flow, $\vec{V}_{f}$, is used to track the location of the saturation time within the porous medium by adopting a Volume-of-Fluid (VOF) approach [2] as it is done for two-phase flows. Thus, the location of the saturation (phreatic) line, $s$, at any time-level can be traced by a transport equation as:

$$
\frac{\partial s}{\partial t}+\left(\vec{V}_{f} \cdot \nabla\right) s=0
$$

And, therefore, the VOF parameter, $s$, can be used to weight the value of the physical properties between the saturated and unsaturated values as:

$$
G=(1-s) G^{u}+s G^{s}
$$

where, for instance, $G^{u}$ is the shear modulus of the unsaturated medium while $G^{s}$ is the shear modulus of the saturated medium.

Notice also that the Navier equation in (1) is assumed to be in steady-state while the Richards equation in (1) is assumed to be in transient mode. This can be implemented in a scheme where the Richards equation is explicitly evolved in time using a time-accurate LCMM scheme as:

$$
p^{k+1}=p^{k}+\frac{\Delta t}{\phi \beta_{f}}\left\{\nabla \cdot\left[\frac{\kappa}{\mu_{f}}\left(\nabla p^{k}+\rho_{f} \vec{g}\right)\right]-\frac{\partial}{\partial t}\left(\nabla \cdot \vec{u}^{k}\right)\right\}
$$

While the deformation field, $\vec{u}$, is updated quasi-statically. Updating the deformation field, $\vec{u}$, need not occur at every time-level but rather every few time-levels to accelerate the solution process. A simple systematic approach can be adopted to determine when the deformation field needs to be updated based on quantified changes of such field.

Furthermore, it is important to note that there is a very significant difference in the time scale at which the pressure signal, $p$, propagates throughout the medium in comparison to the time scale at which the saturation front, $s$, moves within the medium. This is evident in Eqn. (7) where it can be seen that fluid compressibility, $\beta_{f}$, appears in the denominator of the right-hand side. The fact that the fluid compressibility, $\beta_{f}$, is usually a very small number renders the propagation of the pressure signal, $p$, very fast in comparison with the velocity, 
$\vec{V}_{f}$, at which the saturation line, $s$, moves. Therefore, it is very important to take special care when progressing equations (5) and (7) as completely different timesteps, $\Delta t$, must be used. A simple solution to this issue is to solve the Richards equation in (1) as a steady-state equation using, for instance, a global GMRes iteration scheme.

\section{Localized collocation meshless method (LCMM)}

The Meshless formulation begins by defining a set of data centers, $N C$, comprised of points on the boundary, $N B$, and points in the interior, $N I$. These data centers will serve as collocation points for the localized expansion of the different field variables in the domain, $\Omega$, and on the boundary, $\Gamma$, see Figure 1 . The essential difference between boundary points and internal points is simply that boundary conditions will be applied at the first while governing equations will be applied at the latter.

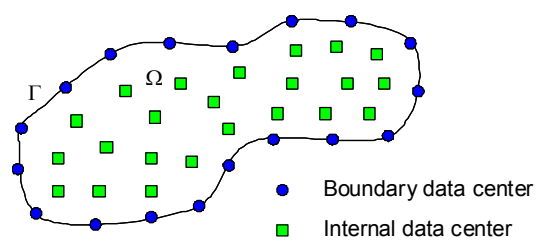

Figure 1: $\quad$ Scattered point distribution in a generalized domain.

The diffusion equation for a general field variable, $\phi$, in a generalized coordinate system, $x$, time, $t$, and a general diffusion coefficient, $\kappa$, is taken as a model governing equation valid in the domain, $\Omega$ :

$$
\frac{\partial \phi}{\partial t}(x, t)=\kappa \nabla^{2} \phi(x, t)
$$

In addition, a set of generalized boundary conditions for the variable, $\phi$, on the boundary, $\Gamma$, are given by:

$$
\hat{\beta}_{1} \frac{\partial \phi}{\partial n}+\hat{\beta}_{2} \phi=\hat{\beta}_{3}
$$

where $\hat{\beta}_{1}, \hat{\beta}_{2}$, and $\hat{\beta}_{3}$ are imposed coefficients that dictate the boundary condition type and constrain values. A linear localized expansion over a group or topology of influence points, $N F$, around each data center is sought such that:

$$
\phi(x)=\sum_{j=1}^{N F} \alpha_{j} \chi_{j}(x)+\sum_{j=1}^{N P} \alpha_{j+N F} P_{j}(x)
$$


The terms $\alpha_{j}$ represent the unknown expansion coefficients while the terms $\chi_{j}(x)$ are expansion functions defined a-priori. $N P$ is a number of additional polynomial functions, $P_{j}(x)$, added to the expansion to guarantee that constant and linear fields can be retrieved by the expansion exactly. Notice that the time dependency has been dropped as a different expansion will be performed for each time level and, therefore, the expansion coefficients, $\alpha_{j}$, will vary as time progresses. The expansion functions, $\chi_{j}(x)$, may be selected from the family of Radial-Basis functions (RBF). Such functions consist of algebraic expressions uniquely defined in terms of the Euclidean distance, $r_{j}(x)$, from a general field point, $x$, to an expansion point, $x_{j}$, and we specifically utilize the inverse Multiquadric RBF as the expansion functions of choice: $\chi(x)=\left[r(x)^{2}+c^{2}\right]^{-1 / 2}$.

The selection of an influence region or localized topology of expansion around each data center is easily accomplished by a circular (spherical in 3D) search around each data center. The search is automated to guarantee that a minimum number of points will be included and additional criteria, such as including all directions around internal data centers, are met. In addition, this search must guarantee that topologies around boundary data centers do not include opposing boundaries or points around a re-entry corner. Figure 2 shows typical collocation topologies for internal and boundary data centers including re-entry corners and opposing boundaries and the circular search to build the topology around an internal data center of a typical non-uniform point distribution. The collocation of the known field variable, $\phi$, (from the previous time level or iteration step) at the points within the localized topology, leads to the following in matrix-vector form:

$$
\{\phi\}=[C]\{\alpha\}
$$
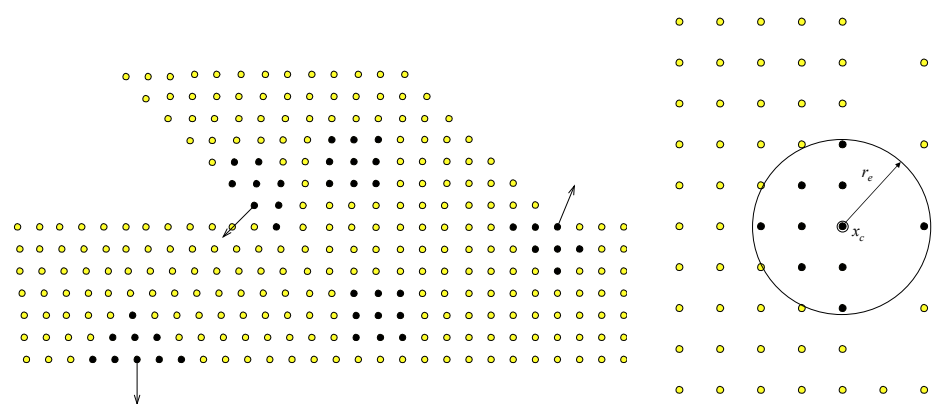

Figure 2: Collocation topology for internal, boundary, corner data centers and topology selection on a non-uniform point distribution. 
And, therefore, the expansion coefficients can be determined as: $\{\alpha\}=[C]^{-1}\{\phi\}$ and the collocation matrix and the known vector are:

$$
[C]=\left[\begin{array}{cccccc}
\chi_{1}\left(x_{1}\right) & \cdots & \chi_{N F}\left(x_{1}\right) & P_{1}\left(x_{1}\right) & \cdots & P_{N P}\left(x_{1}\right) \\
\vdots & \ddots & \vdots & \vdots & \ddots & \vdots \\
\chi_{1}\left(x_{N F}\right) & \cdots & \chi_{N F}\left(x_{N F}\right) & P_{1}\left(x_{N F}\right) & \cdots & P_{N P}\left(x_{N F}\right) \\
P_{1}\left(x_{1}\right) & \cdots & P_{1}\left(x_{N F}\right) & 0 & \cdots & 0 \\
\cdots & \ddots & \cdots & \vdots & \ddots & \vdots \\
P_{N P}\left(x_{1}\right) & \cdots & P_{N P}\left(x_{N F}\right) & 0 & \cdots & 0
\end{array}\right]_{N F+N P, N F+N P} \quad\{\phi\}=\left\{\begin{array}{c}
\phi\left(x_{1}\right) \\
\vdots \\
\phi\left(x_{N F}\right) \\
0 \\
\vdots \\
0
\end{array}\right]_{N F+N P, 1}
$$

A simple optimization search is employed to determine the value of the inverse Multiquadric RBF shape parameter, $c$, used in every expansion over the different local topologies that cover the entire field. An initial guess for $c$ is based on the ratio of the average distance between data centers in a topology to the number of points in the topology. A line search is performed to slightly modify the value of the shape parameter, $c$, until the resulting collocation matrix, $[C]$, yields a condition number in the range between $10^{11}$ and $10^{12}$ (in doubleprecision). This range of condition number for the collocation matrix, $[C]$, has been documented to produce interpolations that render smooth derivative fields for a wide range of test functions. It is important to mention that the resulting collocation matrix, $[C]$, depends only on the geometrical distribution of the points within each localized topology and therefore, the optimization of the shape parameter, $c$, is performed at a setup stage before the solution process begins. However, there may be instances when running-time optimization of the shape parameter, $c$, may be necessary as, for example, when adaptive refinement is performed or when sharp discontinuities in the solution field are found.

The derivatives of the field variable are calculated at the data center, $x_{c}$, of each topology, for any linear differential operator, $L$, can be applied over the localized expansion equation as:

$$
L \phi\left(x_{c}\right)=\sum_{j=1}^{N F} \alpha_{j} L \chi_{j}\left(x_{c}\right)+\sum_{j=1}^{N P} \alpha_{j+N F} L P_{j}\left(x_{c}\right)
$$

Or, in matrix-vector form, $L \phi_{c}=\left\{L_{c}\right\}^{T}\{\alpha\}$, and upon introducing the expansion coefficients, $\{\alpha\}$, leads to:

$$
L \phi_{c}=\{L\}^{T}\{\phi\}
$$

where $\{L\}^{T}=\left\{L_{c}\right\}^{T}[C]^{-1}$. The coefficients of the vector $\{L\}$ of size $(N F, 1)$ directly interpolate any differential operation of the field variable distribution, $\{\phi\}$, at the data center of the topology. Therefore, evaluation of the field variable derivatives at everyone of the data centers is provided by a simple inner product of two small vectors, $\{L\}$, which can be pre-built and stored, and $\{\phi\}$ 
which is the updated field variable distribution in the topology of the data center. Applying to the generalized diffusion equation, using a first-order finitedifferencing leads to:

$$
\phi_{c}^{k+1}=\phi_{c}^{k}+\kappa \Delta t\left(\nabla^{2} \phi_{c}^{k}\right) \Rightarrow \phi_{c}^{k+1}=\phi_{c}^{k}+\kappa \Delta t\{L\}^{T}\{\phi\}^{k}
$$

where the superscript, $k$, denotes the time level and $\Delta t$ denotes the size of the time step. The solution field, $\{\phi\}$, at every point at the previous time level, $k$, can very efficiently yield the updated field variable value at each data center, $x_{c}$, through a simple inner product of small vectors. Similarly, we find:

$$
\left.\hat{\beta}_{1} \frac{\partial \phi}{\partial n}\right|_{x_{c}}+\hat{\beta}_{2} \phi_{c}=\hat{\beta}_{3} \Rightarrow \hat{\beta}_{1}\{\partial n\}^{T}\{\phi\}+\hat{\beta}_{2} \phi_{c}=\hat{\beta}_{3}
$$

where the interpolation vector, $\{\partial n\}$, yields the normal derivative of the field variable, $\{\phi\}$, at the boundary topology data center, $x_{c}$. Ultimately, through some algebraic manipulation, a simple relation can be arrived at to determine the boundary field variable at the current time level, $k+1$, as: $\phi_{c}^{k+1}=\{\Gamma\}^{T}\{\phi\}^{k}$ where the boundary interpolation vector, $\{\Gamma\}$, is composed by a combination of the normal derivative interpolation vector, $\left\{\partial_{n}\right\}$, and the boundary condition coefficients, $\hat{\beta}_{1}, \hat{\beta}_{2}$, and $\hat{\beta}_{3}$, and, therefore, can be pre-built at a setup stage for every boundary data center, $x_{c}$. The normal derivative interpolation vector, $\{\partial n\}$, may be pre-built in one of two ways. A simple approach is to express this vector as a combination of derivative vectors in all directions times their corresponding unit normal vector components. For instance, in 3D Cartesian coordinates, this is: $\{\partial n\}=\{\partial x\} n_{x}+\left\{\partial y n_{y}+\{\partial z\} n_{z}\right.$. A slightly more involved but more stable approach is to generate additional internal points that "shadow" each boundary point in the direction of the normal vector into the domain, $\Omega$, and use these shadow points to directly approximate the normal derivatives at each boundary data center, see Figure 3.

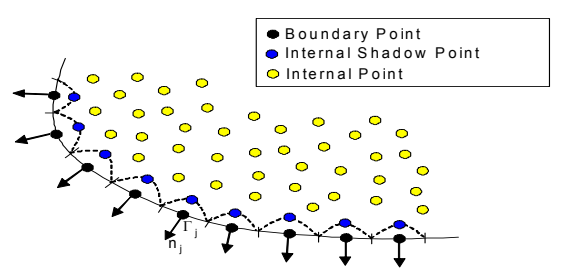

Figure 3: Internal shadow points to compute normal derivatives.

The shadow point approach will mitigate the inherent inaccuracies of the directional derivative interpolation vectors of the truncated topologies of boundary data centers especially of those around corners and highly curved 
boundaries. Following this approach, the normal derivative interpolation vector is, $\{\partial n\}=\left\{1 / r_{s}, 0 \ldots 0,-1 / r_{s}, 0 \ldots 0\right\}^{T}$, where $r_{s}$ is the distance from the boundary data center to its corresponding internal shadow point. Notice that all of the elements of the interpolation vector, except two, vanish. Additionally, higher order differentiation can be accomplished by simply inserting multiple rows of internal shadow points in the normal direction of each boundary data center.

The LCMM reduces the burden of the more common global interpolation methods by expanding the field variable locally around each data center to obtain its derivatives that are then used in time-marching or iterative schemes. This approach generates multiple but small interpolation matrices rather than the large and fully-populated global interpolation matrix of the standard global interpolation methods. However, since the approach relies on expanding known values of the field variables, it is applicable to explicit time-marching schemes and inapplicable directly to steady problems. Moreover, time marching can always be considered as a relaxation scheme for the iterative solution of steadystate problems. We also utilize upwinding schemes for convectively dominated flows utilizing the concept of "Virtual Points", see Figure 4.

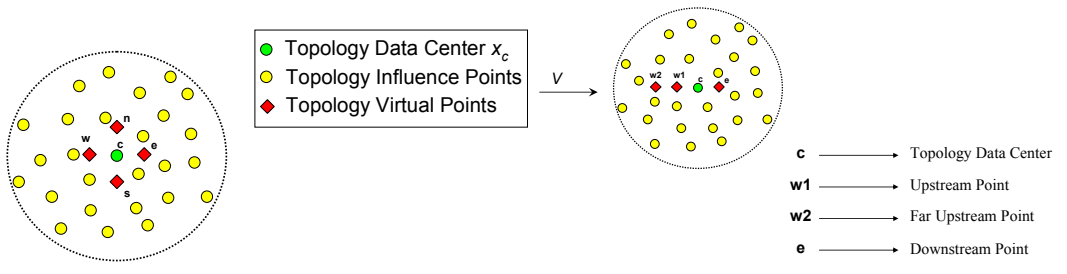

Figure 4: Upwinding with "Virtual Points" around the data center, $x_{c}$.

\section{The levee problem}

The problem of water flow through a compacted sand poro-elastic levee combines the effects of storm surges to the levee as well as the progressive piping (crack propagation) within the levee due to the pore pressure and stress field. The schematics of this problem are illustrated in Figure 5. The trapezoidal portion of the levee is $40 \mathrm{~m}$ at the base and $3 \mathrm{~m}$ at the top with a height of $6 \mathrm{~m}$. The water level on the left-hand side of the levee is $1 \mathrm{~m}$ from the top of the levee. A $3 m$-long pipe (crack) inclined $45^{\circ}$ is included $8 m$ from the left-hand

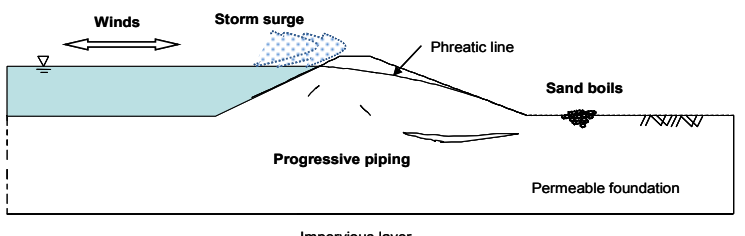

Figure 5: Groundwater flow through a poro-elastic sand levee problem. 


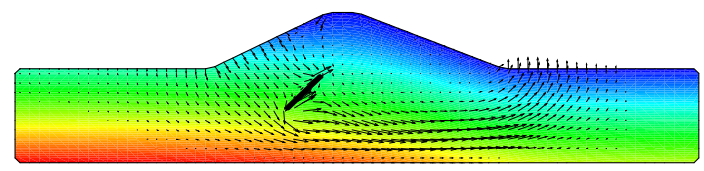

Figure 6: Hydrostatic pressure levels and water flow vectors within the levee. corner of the trapezoidal section of the levee. The levee hydraulic conductivity is $K_{h}=10^{-7} \mathrm{~m} / \mathrm{s}$, the Poisson ratio $\nu=0.35$, the modulus of elasticity $E=120 M P a$, the dry density $\rho=1500 \mathrm{~kg} / \mathrm{m}^{3}$, the specific gravity of soil particles $S G=2.65$, the porosity $\phi=0.3$, the water density, viscosity, and compressibility are $\rho_{f}=1000 \mathrm{~kg} / \mathrm{m}^{3}, \mu_{f}=10^{-3} \mathrm{~Pa} \cdot \mathrm{s}$, and $\beta_{f}=4.55 \cdot 10^{-10} \mathrm{~m}^{2} / \mathrm{N}$ respectively. The contour plots of hydrostatic pressure levels and water flow vectors within the levee and around the pipe are shown in Figure 6 after a steadystate solution is achieved. The time progression of the saturation front within the poro-elastic levee at $t=20,40,60,80,100,120$ days is shown in Figure 7 .
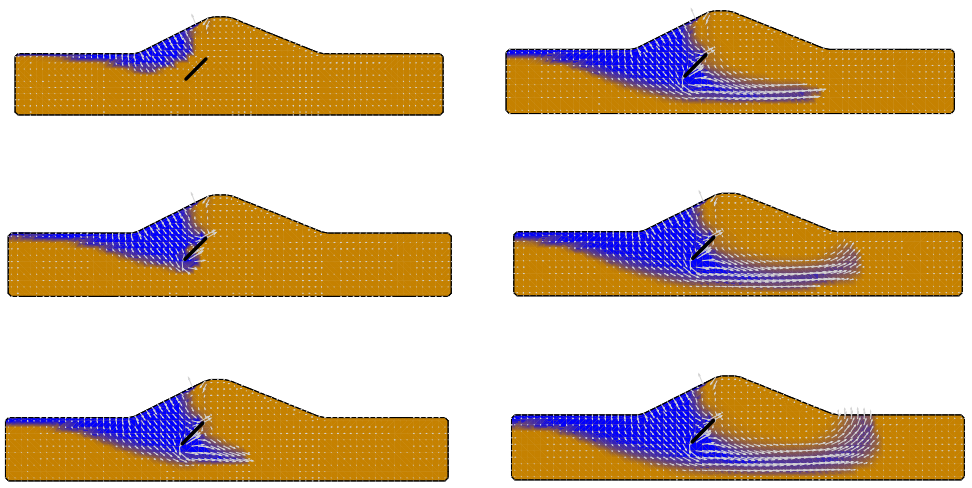

Figure 7: Time progression of the saturation front: 20, 40, 60, 100 and 120 days.

\section{Conclusions}

We applied the LCCM to poro-elastic flow in a levee resolving the interaction between the water flow and soil in a time-accurate manner. The method shows promise in applications to planned future studies of the piping problem.

\section{References}

[1] Bonelli, S., Brivois, O., Borghi, R., and Benahmed, N. (2006). "Observation, analysis and modeling in complex fluid media - On the modeling of piping erosion." C. R. Mecanique, 334: 555-559. 
[2] Bonelli, S., and Brivois, O (2008). "The scaling law in the hole erosion test with a constant pressure drop." International Journal for Numerical and Analytical Methods in Geomechanics, 32:1573-1595.

[3] Chen, C., Liu, J., Xia, J., Li, Z. (2006). "Integrated geophysical techniques in detecting hidden dangers in river embankments." Journal of Environmental and Engineering Geophysics, 11(2): 83-94.

[4] El Shamy, U., and Aydin, F. (2008), "Multi-scale modeling of floodinduced piping in river levees", Journal of Geotechnical and Geoenvironmental Engineering, 134 (9): 1385-1389.

[5] Evans, J. E., Scuddkr, D. M., Gottgens, J. F., and Gill, W. M. (2000). "Lessons from a Dam Failure." Ohio Journal of Science, 100(5):121-131.

[6] Foster, M., Fell, R., and Spannagle, M. (2000). "The statistics of embankment dam failures and accidents." Canadian Geotechnical J., 37:1000-1024.

[7] Gattinoni, P., and Francani, V. (2009). "A Tool for Modeling Slope Instability Triggered by Piping." World Academy of Science, Engineering and Technology, 56: 471-477.

[8] Hagerty, D.J. (1991). "Piping/sapping erosion. I: basic considerations." Journal of Hydraulic Engineering, 117(8): 991-1008.

[9] Hagerty, D.J. (1991). "Piping/sapping erosion. II: identification-diagnosis." Journal of Hydraulic Engineering, 117(8): 1009-1025.

[10] Hung, M.-H., Lauchle, G. C., and Wang, M.C. (2009). "Seepage-Induced Acoustic Emission in Granular Soils." Jl. of Geotechnical and Geoenvironmental Engineering, 135(4):566-572.

[11] ICOLD (1995). Dam Failures - Statistical Analysis. Bulletin 99, International Commission on Large Dams, Paris, France, pp. 30-31.

[12] Inazaki, T. (2007). "Integrated geophysical investigation for the vulnerability assessment of earthen levee." Proc. Symp. on the Appln. of Geophysics to Environmental and Eng.Problems (SAGEEP), Denver, CO.

[13] Johansen, P.M., and Eikevik, J.H. (1997). "Internal erosion and rehabilitation of Jukla rockfill dams." Intl. Congress on Large Dams, 19(2):245-253.

[14] Kakuturu, S., and Reddi, L. N. (2006). "Evaluation of the Parameters Influencing Self-Healing in Earth Dams."ASCE Jl. of Geotechnical and Geoenvironmental Engg., 132(7): 879-889.

[15] Kakuturu, S., and Reddi, L. N. (2006). "Mechanistic Model for SelfHealing of Core Cracks in Earth Dams."ASCE Jl. of Geotechnical and Geoenvironmental Engg., 132(7): 890-901.

[16] Lakshmikantha, M.R., Prat, P.C., Ledesma, A. (2006). "An experimental study of cracking mechanisms in drying soils." 5th ICEG Environmental Geotechnics: Opportunities, Challenges and Responsibilities, Vol.I: 533540.

[17] Mansur, C.I., Postol, G., and Salley, J.R. (2000), "Performance of relief well systems along Mississippi River levees", J. Geotechnical and Geoenvironmental Engineering, 126 (8): 727-738. 
[18] Ojha, C.S.P., Singh, V.P., Adrian, D.D. (2003), "Determination of critical head in soil piping", Journal of Hydraulic Engineering, 129(7): 511-518.

[19] Ozkan, S., Adrian, D. D., Sills, G. L., and Singh, V.P., (2008). "Transient Head Development due to Flood Induced Seepage under Levees." ASCE J. of Geotechnical and Geoenvironmental Engineering, 134(6): 781-789.

[20] Sellmeijer, J. B., and Koenders, M. A. (1991). "A mathematical model for piping.' Applied Mathematical Modeling, 15(6): 646-651.

[21] Sjödahl, P. (2006). Resistivity investigation and monitoring for detection of internal erosion and anomalous seepage in embankment dams. Doctoral Thesis, Engineering Geology, Lund University, Sweden.

[22] Sills, G.L., Vroman, N.D., Wahl, R.E., and Schwanz, N.T. (2008), "Overview of New Orleans levee failures: Lessons learned and their impact on national levee design and assessment", Journal of Geotechnical and Geoenvironmental Engineering, 134 (5): 556-565.

[23] Vallejo, L.E. (1993). "Shear stress and the hydraulic fracturing of earth dam soils." Soils and Foundations, 33(3): 14-27.

[24] Watts, R., Burk, K., McLaren, M., Wolfe, J., Zender, K. (2002). "Failure of the Teton dam: Geotechnical aspects." Intl. Water Power and Dam Construction, 54(7): 30-31.

[25] Yi C., Wang B., Jin M., and Guo, Z. (2007), “Two-dimensional simulation of underground seepage in a dangerous piping zone of the Jingjiang Great Levee, the middle reach of the Yangtze River," Quarterly $\mathrm{Jl}$. of Engineering Geology and Hydrogeology, 40 (1): 85-92.

[26] Zaslavsky, D., and Kassiff, G. (1965). "Formulation of piping mechanism in cohesive soils." Geotechnique, 15(3): 305-316.

[27] Zhang, L.M., and Chen, Q. (2006). "Seepage failure mechanism of the Gouhou rockfill dam during reservoir water infiltration." Soils and Foundations, 46(5): 557-568.

[28] Divo, E. and Kassab, A.J., (2007), “An Efficient Localized RBF Meshless Method for Fluid Flow and Conjugate Heat Transfer," ASME J. of Heat Transfer, 129: 124-136.

[29] Divo, E. and Kassab, A.J., (2008), "Localized Meshless Modeling of Natural Convective Viscous Flows," Numerical Heat Transfer, Part A: Fundamentals, 53 (6): 487-509.

[30] El Zahab, Z., Divo, E., and Kassab, A.J., (2009), “A Localized Collocation Meshless Method (LCMM) for Incompressible Flows CFD Modeling with Applications to Transient Hemodynamics," Engineering Analysis with Boundary Elements, 33 (8-9):1045-1061.

[31] El Zahab, Z., Divo, E., and Kassab, A.J., (2009), “A Meshless CFD Approach for Evolutionary Shape Optimization of Bypass Grafts Anastomoses," Inverse Problems in Science and Engineering, 17(3): 411435.

[32] El-Zahab, Divo, E., and Kassab, A.J, (2010), "Minimization of the Wall Shear Stress Gradients in Bypass Grafts Anastomoses using Meshless CFD and Genetic Algorithms Optimization" Comp. Mthds, in Biomech. and Biomed. Engg, 13(1): 35-47. 